\title{
Postoperative Changes in Alignment in Patients Surgically Corrected for Esotropia and Exotropia in a Tertiary Government Hospital
}

\author{
Ruth R. Peña, Marissa N. Valbuena, Alvina Pauline D. Santiago and Andrea Kristina M. Pajarillo \\ Department of Ophthalmology, College of Medicine and Philippine General Hospital, University of the Philippines Manila
}

\begin{abstract}
Objective. To compare the postoperative alignment of patients surgically corrected for esotropia or exotropia 6 weeks and 6 months after surgery.

Method. This retrospective study reviewed clinical records of patients who underwent horizontal muscle surgery at the University of the Philippines-Philippine General Hospital from 2010 to 2014 . The changes in alignment from 1 week to 6 weeks and from 1 week to 6 months after surgery were compared for overcorrection, undercorrection, and adequate correction groups. ANOVA and Pearson correlation were used.

Results. Twenty two esotropia and 10 exotropia patients were included. Overcorrected esotropia patients had an esotropic change in alignment (15.50+13.44 PD after 6 weeks, $p=0.026 ; 25+18.38$ PD after 6 months, $p=0.008)$. Under corrected esotropia patients had an exotropic change in alignment $(-1.25+5.91$ PD after 6 weeks, $p=0.026 ;-4.38+14.16$ PD after 6 months, $p=0.008)$. Undercorrected exotropia patients had an esotropic change in alignment $(6.67+6.35$ PD after 6 weeks, $p=0.028 ; 6+9.85$ PD after 6 months, $p=0.024)$. The presence of vertical deviations in esotropia caused an exotropic change in alignment after 6 months $(p=0.03)$.
\end{abstract}

Conclusion. Although an exotropic drift is more commonly reported, fusional vergences may account for postoperative alignment changes towards orthotropia.

Key Words: esotropia, exotropia, postoperative strabismus

\section{INTRODUCTION}

Strabismus surgical success rates range from $60 \%$ to $85 \%^{1-3}$, thus patients are advised that more than one surgery may be needed to correct ocular deviations. ${ }^{4,5}$ In this tertiary government hospital, it has been observed that secondary operations for strabismus are few despite a number of cases not achieving optimal alignment after surgery. Some strabismus surgeons plan reoperations after the full healing period of six weeks because alignment can still change dramatically during this time. Additional observation is also warranted after this period if alignment progressively improves over time. ${ }^{4}$

Exotropia surgery is usually associated with recurrence and Visual Sciences Conference November 29,2016, Dr. Romeo B. Espiritu Auditorium, $5^{\text {th }}$ floor, Sentro Oftalmologico Jose Rizal Building, Philippine General Hospital.

Corresponding author: Ruth R. Peña, MD

Department of Ophthalmology

Philippine General Hospital

University of the Philippines Manila

Taft Avenue, Manila 1000, Philippines

Email: pena_rr@yahoo.com

of the exodeviation. ${ }^{5}$ Some strabismus surgeons advocate initial postoperative overcorrection to counteract this exotropic drift. ${ }^{6}$ There is no consensus however, as to the amount of overcorrection needed to come up with a good long term postoperative alignment. According to Ruttum, initial postoperative overcorrection is desirable but does not ensure a good outcome. ${ }^{7}$ 
Postoperative Changes in Alignment in Strabismus

Pineles et al. also noted that the amount of esotropic overcorrection in intermittent exotropia patients did not predict long term outcome stability and consecutive esotropia. ${ }^{8}$ Yam et al. ${ }^{9}$ and Park and $\mathrm{Kim}^{10}$ noted larger exotropic drifts in patients with larger initial overcorrections for exotropia. Park and Kim also noted larger exotropic drifts in patients with larger preoperative exodeviations. ${ }^{10}$ Mireskandari et al. showed that orthotropia to 8 prism diopters (PD) esotropia in the immediate postoperative period was associated with successful exotropia surgery. ${ }^{11}$

Unlike exotropia surgery, esotropia surgery does not usually take into account the exotropic drift, hence there are no studies suggesting immediate postoperative target alignments. The study by Mireskandari et al. showed that achieving orthotropia to 8 PD esotropia for exotropia cases resulted in successful surgery. This effect did not apply to esotropia cases. ${ }^{11} \mathrm{~A}$ study by Chatzistefanou et al. on long term outcomes of patients with large angle infantile esotropia who underwent 3 horizontal muscle surgery showed a trend towards an exotropic drift after surgery. This occurred more often than an esotropic drift, especially in patients with smaller preoperative esodeviations. ${ }^{12}$ In a study by Maruo et al., infantile and acquired esotropia cases showed a tendency to become exotropic four years after surgery. Maruo et al. recommended correction to orthophoria or minimal esotropia to obtain optimal alignment. ${ }^{13}$

Other factors affecting postoperative success for esotropia and exotropia have been studied. These include effects of the age at onset of strabismus, ${ }^{8,14-16}$ age at surgical intervention, $, 16,17$ preoperative deviation, ${ }^{8,9,12,18}$ type of strabismus, ${ }^{8,17}$ amblyopia, ${ }^{12,14-16}$ and associated vertical deviations. ${ }^{12,16,17}$ Results were varied. According to Egbert and Fantin, the strongest determinants of response to surgery are the types of strabismus and preoperative deviation. ${ }^{19}$

Previous studies showed that long term postoperative outcomes varied depending on the length of the follow-up period. This study aimed to determine the changes in deviation over a period of 6 weeks and of 6 months. This would help determine which preoperative and postoperative characteristics would show small or large drifts over time and the direction of these drifts (exotropic or esotropic). It had been the first author's observation that the usual postoperative complication of strabismus surgery in this institution during the first few months was undercorrection. Although patients were advised a possible reoperation even during the initial counseling, subsequent follow-ups usually showed improvement in ocular alignment. With patients in this institution rarely undergoing secondary strabismus surgery presumably due to improvement in alignment over time, it would be beneficial to determine the amount of change in deviation over time in patients who underwent strabismus surgery, whether they were overcorrected, undercorrected, or adequately corrected for strabismus. In a setting of a tertiary training government hospital where preoperative examinations and surgeries are done by different ophthalmologists, individual differences in examination and surgical techniques are inevitable. As a result, consistent surgical results are difficult to achieve. Knowing the postoperative changes in deviation and the factors affecting these would provide insight for surgical planning for future cases. Establishing relationships of preoperative and postoperative characteristics with postoperative alignment would help determine cases needing more meticulous preoperative examinations, surgical techniques, and postoperative monitoring. This would also help in patient education and counselling needed for the social stigma, surgical risks and costs of strabismus.

The purpose of this study was to compare the change in the postoperative ocular alignment at 6 weeks and at 6 months after surgery in patients who were initially surgically undercorrected, overcorrected, or adequately corrected for esotropia or exotropia. It also aimed to determine if preoperative characteristics such as esotropia or exotropia type, age at onset of strabismus, age at strabismus surgery, vertical deviations, amblyopia, and preoperative deviation were correlated to these changes in ocular alignment.

\section{METHODS}

Clinical records of all patients who underwent muscle surgery for esotropia or exotropia with sufficient followup time after surgery in the University of the PhilippinesPhilippine General Hospital's Sentro Oftalmologico Jose Rizal from 2010 to 2014 were reviewed. Included were patients who underwent one to four horizontal muscle surgery for a horizontal deviation, with or without vertical muscle surgery, with or without adjustable sutures and who completed at least a 6 month follow-up period. Excluded were patients with paralytic, restrictive or neurologic strabismus, patients with other ocular pathologies, patients with previous strabismus surgery, and patients who did not complete the 6 month minimum follow-up period. Patients with incomplete chart data were not included in the study. Surgeries and preoperative and postoperative measurements of alignment were done by different ophthalmologists. The alternate prism and cover test was used to measure the amount of deviation, when applicable.

The type of esotropia or exotropia, age at onset of strabismus, age at strabismus surgery, presence of vertical deviations, presence of amblyopia, and preoperative deviation were noted. Esotropia cases were classified as infantile, accommodative, or partially accommodative. Exotropia was classified as infantile, intermittent (with episodes of orthophoria), or constant (without orthophoric episodes). Presence or absence of vertical deviations was noted. Vertical deviations included inferior oblique overactions and dissociated vertical deviations. Presence or absence of amblyopia was noted. Amblyopia was defined as a visual acuity difference of more than two lines between the two eyes, a bilateral best corrected visual acuity worse than 
20/40 for patients 7 years old and above or a bilateral best corrected visual acuity worse than the age appropriate visual acuity for children less than 7 years of age., ${ }^{5,20}$ Fixation behavior visual acuity for preverbal and illiterate patients was converted to the corresponding linear letter optotype visual acuity equivalent. ${ }^{21}$ Visual acuity was converted to $\log M a r$ equivalent for analysis. Preoperative deviation was grouped into $\geq 50$ prism diopters (PD) (large angle) or $<50 \mathrm{PD}$.

Esotropia and exotropia cases were grouped separately. Patients were grouped according to the 1 week postoperative alignment as overcorrected ( $>10 \mathrm{PD})$, undercorrected (>10PD), and adequately corrected (within +/-10PD). The change in alignment from 1 week to 6 weeks after surgery and from 1 week to at least 6 months after surgery was noted for the different groups. A change in alignment to esodeviation was assigned a positive $(+)$ sign and a change in alignment to exodeviation was assigned a negative (-) sign. The postoperative change in alignment at 6 weeks and at $\geq 6$ months was also compared according to the different preoperative factors of esotropia or exotropia type, age at onset of strabismus, age at strabismus surgery, presence of vertical deviations, presence of amblyopia and preoperative deviation.

Descriptive statistics were used to show patient characteristics and postoperative alignment outcomes. Descriptive statistics were also used to show the direction of change in postoperative alignment (exotropic or esotropic drift) among the patients. A Pearson correlation coefficient (Microsoft Excel 2010) was used to determine the correlation of age at onset of strabismus and age at strabismus surgery with postoperative change in alignment. ANOVA (SPSS version 20) was used to determine a significant difference in the mean change in alignment at the 6 week and at $\geq 6$ month follow-up periods of the three immediate postoperative groups of overcorrection, undercorrection, and adequate correction. Patients were also classified according to different preoperative characteristics. ANOVA was used to determine a significant difference in the change in alignment of strabismus patients when classified according to the different preoperative characteristics. A p value of $\leq 0.05$ was statistically significant.

\section{RESULTS}

\section{Esotropia}

The preoperative characteristics of esotropia patients are summarized in Table 1.The mean postoperative alignment of esotropia patients was $2 \pm 10.84 \mathrm{PD}$ esotropia (ET) at 1 week, $4.53 \pm 8.5 \mathrm{PD} \mathrm{ET}$ at 6 weeks and $5.43 \pm 9.81 \mathrm{PD} \mathrm{ET}$ at $\geq 6$ months. The mean change in alignment of esotropia patients from 1 week to 6 weeks $(2.53 \pm 7.72 \mathrm{PD})$ and from 1 week to $\geq 6$ months $(3.43 \pm 11.86 \mathrm{PD})$ after surgery was in an esotropic or inward direction. Comparison of changes in alignment at 6 weeks and at $\geq 6$ months for different preoperative and postoperative characteristics are shown
Table 1. Preoperative characteristics of esotropia patients

\begin{tabular}{lc} 
Esotropia (n=22) & $\mathbf{n}, \%$ \\
Gender & $11(50 \%)$ \\
Male & $11(50 \%)$ \\
Female & $2.08 \pm 2.13$ years (range 0-7) \\
\hline Mean age at strabismus onset & $11.59 \pm 9.57$ years (range 2-41) \\
\hline Mean age at strabismus surgery & $10(45 \%)$ \\
\hline Esotropia Type & $7(32 \%)$ \\
Infantile & $5(23 \%)$ \\
Decompensated Accommodative & \\
Partially Accommodative & $9(41 \%)$ \\
\hline Presence of vertical deviations & $13(59 \%)$ \\
With vertical deviations & $4(18 \%)$ \\
Without vertical deviations & $18(82 \%)$ \\
\hline Presence of Amblyopia & $53.94 \pm 23.47$ PD \\
With amblyopia & \\
Without amblyopia & $10(45 \%)$, mean $=32 \pm 8.15$ PD \\
\hline Mean preoperative deviation & $12(55 \%)$, mean=72.22 \\
\hline Size of preoperative deviation & \\
$<50$ PD &
\end{tabular}

PD - Prism diopters

in Table 2. There was a significant difference in the mean change in alignment of the overcorrected, undercorrected, and adequately corrected groups after 6 weeks $(p=0.026)$ and after $\geq 6$ months $(p=0.008)$. Overcorrected and adequately corrected groups had an esotropic drift while the undercorrected group had an exotropic drift. There was no significant difference in the postoperative alignment of the esotropia patients when grouped according to esotropia type, presence of amblyopia, and preoperative deviation. The presence of vertical deviations in esotropia was associated with an exotropic change in alignment after 6 months $(p=0.03)$. There was no correlation between the age at onset of esotropia and the change in postoperative alignment after 6 weeks (Pearson=0.15) and after $\geq 6$ months (Pearson=0.28). There was no correlation between the age at strabismus surgery and the change in postoperative alignment after 6 weeks (Pearson=0.23). There was a weak positive relationship between the age at strabismus surgery and the change in alignment after $\geq 6$ months (Pearson $=0.35$ ).

\section{Exotropia}

The preoperative characteristics of exotropia patients are summarized in Table 3. The mean postoperative alignment of exotropia patients was $4.57 \pm 11.16 \mathrm{PD} \mathrm{XT}$ at 1 week, $7 \pm 8.16 \mathrm{PD} \mathrm{XT}$ at 6 weeks, and $7.8 \pm 10.12 \mathrm{PD}$ $\mathrm{XT}$ at $\geq 6$ months. The mean change in alignment from 1 week to 6 weeks $(-2.43 \pm 9.12 \mathrm{PD})$ and from 1 week to $\geq 6$ months $(-3.23 \pm 9.11 \mathrm{PD})$ after surgery was in an exotropic or outward direction. Comparison of changes in alignment at 6 weeks and at $\geq 6$ months for different preoperative and postoperative characteristics are shown in Table 4. There was a significant difference in the mean change in alignment of the undercorrected and adequately corrected groups 
Table 2. Postoperative change in alignment of esotropia patients grouped according to different postoperative and preoperative characteristics

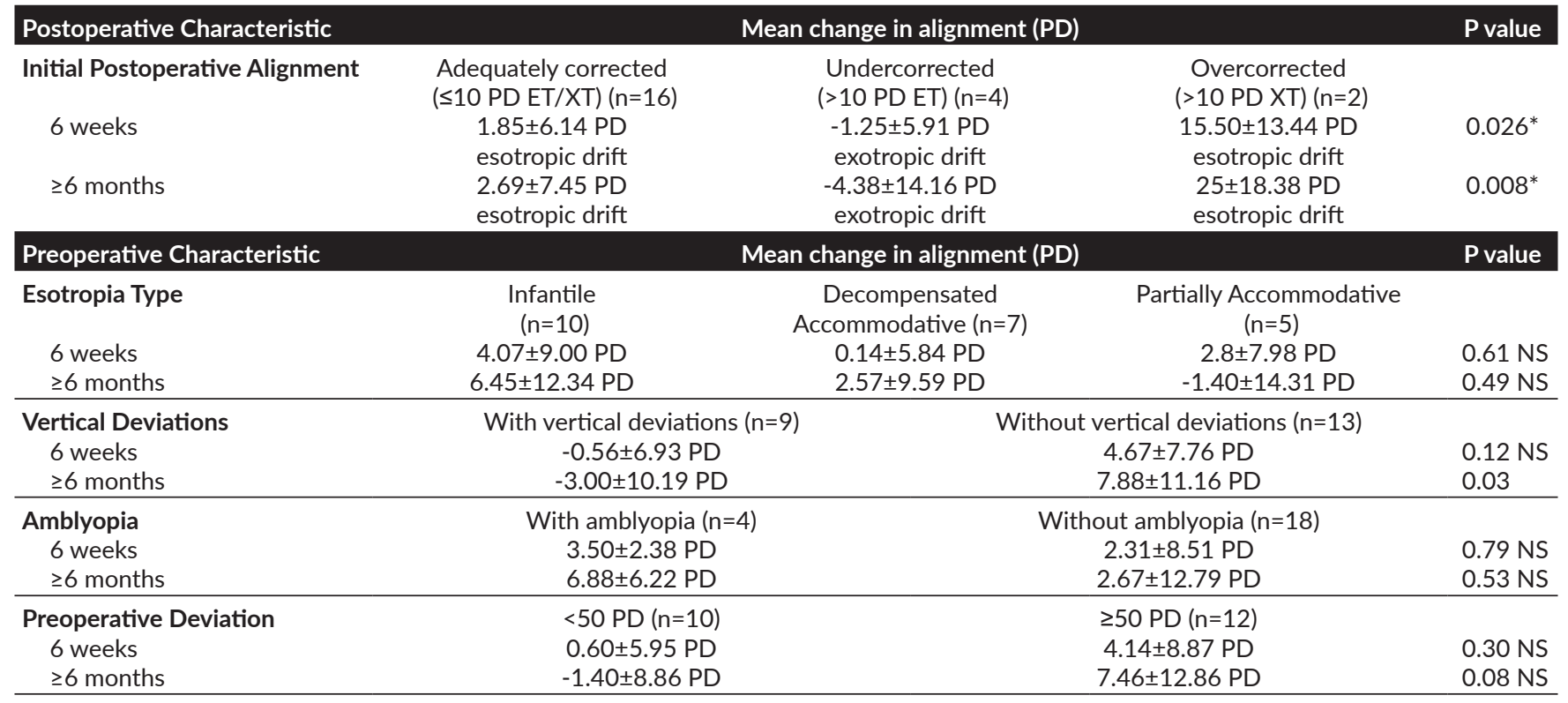

NS - Not significant

Table 3. Preoperative characteristics of exotropia patients

\begin{tabular}{|c|c|}
\hline Exotropia $(n=10)$ & $\mathrm{n}, \%$ \\
\hline $\begin{array}{l}\text { Gender } \\
\text { Male } \\
\text { Female } \\
\end{array}$ & $\begin{array}{l}1(10 \%) \\
9(90 \%) \\
\end{array}$ \\
\hline Mean age at strabismus onset & $5.88 \pm 8.65$ years (range $0-28$ ) \\
\hline Mean age at strabismus surgery & $13.60 \pm 14.35$ years (range $3-50$ ) \\
\hline $\begin{array}{l}\text { Exotropia Type } \\
\text { Infantile } \\
\text { Intermittent } \\
\text { Constant } \\
\end{array}$ & $\begin{array}{l}2(20 \%) \\
5(50 \%) \\
3(30 \%) \\
\end{array}$ \\
\hline $\begin{array}{l}\text { Presence of vertical deviations } \\
\text { With vertical deviations } \\
\text { Without vertical deviations }\end{array}$ & $\begin{array}{l}3(30 \%) \\
7(70 \%) \\
\end{array}$ \\
\hline $\begin{array}{l}\text { Presence of Amblyopia } \\
\text { With amblyopia } \\
\text { Without amblyopia } \\
\end{array}$ & $\begin{array}{l}1(10 \%) \\
9(90 \%) \\
\end{array}$ \\
\hline Mean preoperative deviation & $49.98 \pm 20.14 \mathrm{PD}$ \\
\hline $\begin{array}{l}\text { Size of preoperative deviation } \\
\quad<50 \mathrm{PD} \\
\quad \geq 50 \mathrm{PD}\end{array}$ & $\begin{array}{l}4(40 \%), \text { mean }=30.56 \pm 8.34 \text { PD } \\
6(60 \%), \text { mean }=62.92 \pm 13.64 \text { PD }\end{array}$ \\
\hline
\end{tabular}

$P D$ - Prism diopters

after 6 weeks $(p=0.028)$ and after $\geq 6$ months $(p=0.024)$. The adequately corrected group had an exotropic drift while the undercorrected group had an esotropic drift. There was no significant difference in the postoperative alignment of the exotropia patients when grouped according to exotropia type, presence of amblyopia, and presence of vertical deviations. A preoperative deviation less than 50 $\mathrm{PD}$ was associated with an exotropic change in alignment after 6 weeks $(p=0.02)$. There was no correlation between the age at onset of exotropia and the change in postoperative alignment after 6 weeks (Pearson=0.28) and after $\geq 6$ months (Pearson=0.09). There was no correlation between the age at strabismus surgery and the change in postoperative alignment after 6 weeks (Pearson=0.21) and after $\geq 6$ months (Pearson=0.05 respectively).

\section{DISCUSSION}

\section{Initial postoperative alignment}

Overcorrected esotropia patients were more likely to have an esotropic change in alignment than undercorrected and adequately corrected patients after 6 weeks and after $\geq 6$ months. Undercorrected patients were more likely to have an exotropic change in alignment than overcorrected patients. Adequately corrected patients were less likely to have an esotropic change in alignment than overcorrected patients. A study by Chatzistefanou et al. showed that both exotropic and esotropic changes in alignment occurred in large angle infantile esotropia patients who underwent strabismus surgery. An exotropic drift occurred three times more often than an esotropic drift. ${ }^{12} \mathrm{~A}$ study by Mireskandari et al. showed that the beneficial effect of achieving the target angle in the immediate postoperative period for exotropia patients was not seen in esotropia patients. ${ }^{11}$ These studies showed that postoperative changes in alignment are varied for esotropia. The change in alignment seen in this study was opposite the direction of the postoperative alignment at 1 week, with the overcorrected esotropia patients having an esotropic drift and the undercorrected esotropia patients having an exotropic drift. A study by Maruo et al. showed a tendency for esotropia patients to become exotropic 4 years after surgery. ${ }^{13}$ The longer 4 year follow-up period compared 
Table 4. Postoperative change in alignment of exotropia patients grouped according to different postoperative and preoperative characteristics

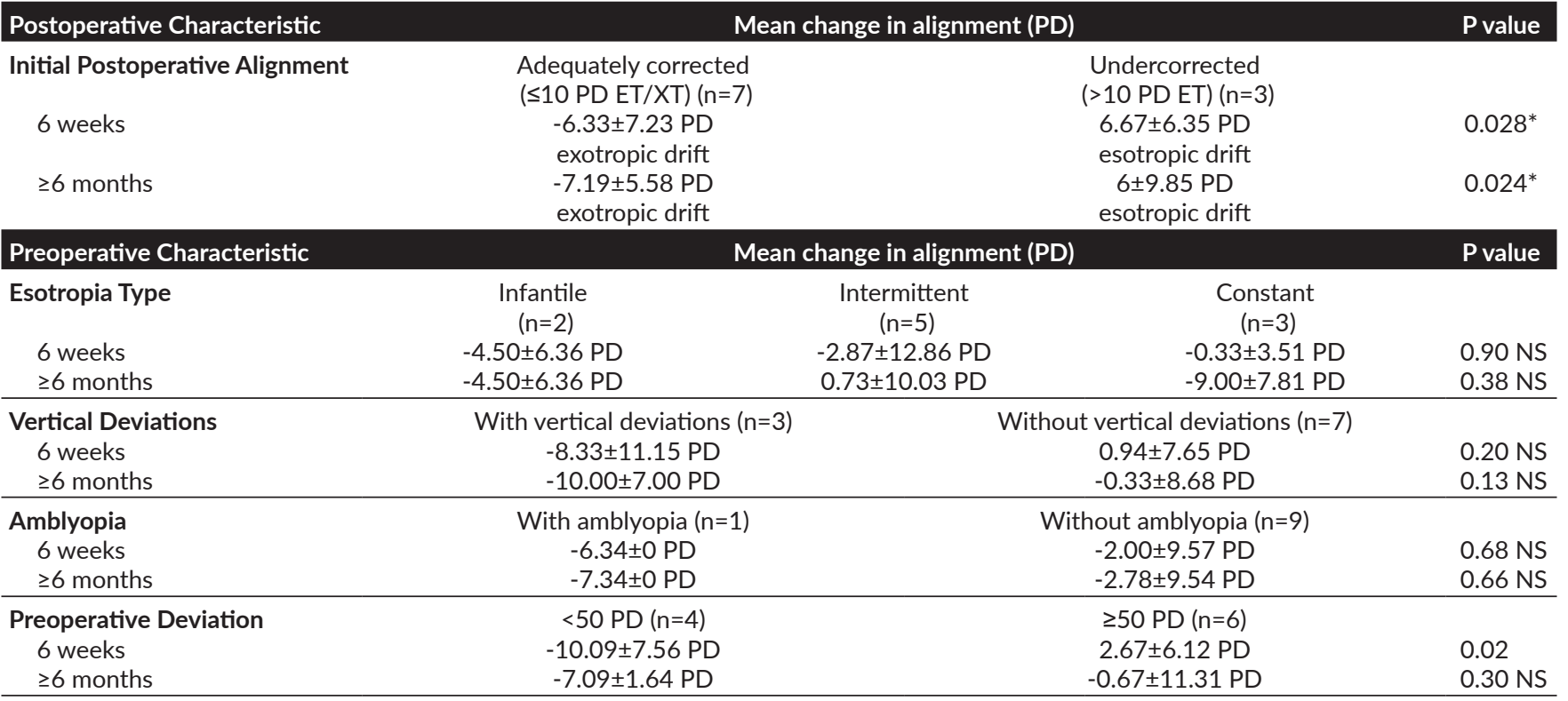

NS - Not significant

to the shorter follow-up period of this study could account for the difference in the results.

Undercorrected exotropia patients were more likely to have an esotropic change in alignment than adequately corrected patients after 6 weeks and after $\geq 6$ months. Adequately corrected exotropia patients were more likely to have an exotropic change in alignment than undercorrected patients. Previous studies showed that larger initial overcorrections were associated with larger postoperative exotropic drifts. ${ }^{7,9,10}$ Although no patients were overcorrected for exotropia in this study, adequately corrected exotropia patients had a significant exotropic drift compared to those who were undercorrected. This supports studies that showed that an exotropic drift happens over time. ${ }^{9,10}$ The undercorrected exotropia patients in this study had a significant tendency to have an esotropic drift compared to the adequately corrected patients. This finding is inconsistent with the usual pattern of an exotropic drift in exotropia patients. A study by Pineles et al. however showed that immediate overcorrection of exotropia did not predict the long term outcome stability or the presence of consecutive esotropia. ${ }^{8}$ These findings are more consistent with the present study.

Overcorrected esotropia patients (exotropic after surgery) had a significant tendency to have an esotropic drift. Undercorrected exotropia patients (exotropic after surgery) also had a significant tendency to have an esotropic drift. This is not the usual exotropic drift expected of inadequately corrected strabismus patients particularly exotropia patients, however this can be seen as a probable attempt of strabismic patients towards fusion. This could also explain the significant tendency of the undercorrected esotropia patients in this study to have an exotropic drift.
Patients who were adequately corrected for exotropia in this study did show an exotropic change in alignment which is consistent with the expected exotropic drift. Although some say that intentional under- or overcorrection is an unreliable strategy because fusional amplitudes may not be sufficient nor steadily exerted, ${ }^{4}$ it seems that in this study, fusional amplitudes were present and used.

\section{Age at onset of strabismus}

There was no correlation between the age at onset of esotropia and exotropia and the change in postoperative alignment.According to Keenan and Willshaw, the age at esotropia onset did not determine a favorable or unfavorable outcome after surgery. ${ }^{15}$ According to Koo et al., the age at onset of exotropia was also not a factor for undercorrection post surgery. ${ }^{16}$ These previous findings support the results of the present study.

\section{Age at strabismus surgery}

There was no correlation between the age at strabismus surgery and the change in postoperative alignment, however there was a weak positive relationship between the age at strabismus surgery and the change in alignment at $\geq 6$ months for esotropia. The later the age at strabismus surgery, the larger the change in postoperative alignment at $\geq 6$ months. This correlation was only a weak one $(\mathrm{P}=0.35)$. Previous studies did not demonstrate the influence of age at strabismus surgery on outcomes of surgery. ${ }^{10,15-17}$ Some say that early surgery provides a better chance for functional improvement. ${ }^{4,5}$ Secondary anatomical changes occur in the ocular structures over time which makes surgical correction at a later date more difficult and less predictable. ${ }^{5}$ 


\section{Strabismus type}

There was no difference in the change in postoperative alignment of esotropia patients grouped according to esotropia type. A study by Maruo et al. showed that there was no difference in the surgical results of esotropia patients grouped according to esotropia type at 1 month, however after 4 years, acquired esotropia cases had better surgical results than the other esotropia types. This was attributed to better binocular vision in acquired ET cases. ${ }^{17}$ Such difference was not seen in this study probably due to the shorter follow-up period.

There was also no difference in the change in postoperative alignment of the exotropia patients grouped according to exotropia type. This result is consistent with the study of Pineles et al. that showed that exotropia type had no effect on the outcome of initially overcorrected exotropia. ${ }^{8}$ Most of the exotropia cases in this study were intermittent or started out as intermittent and were therefore similar with regard to fusional status. Sensory deprivation exotropia patients and patients with neurologic conditions were excluded in this study.

\section{Vertical deviations}

There was no difference between the change in alignment of esotropia patients with vertical deviations and patients without vertical deviations after 6 weeks. After $\geq 6$ months, a significant difference was noted between the 2 groups. The esotropia patients with vertical deviations were more likely to have an exotropic change in alignment after $\geq 6$ months. The studies by Maruo et al. and Mireskandari et al. showed less satisfactory surgical results for patients with esotropia and vertical deviations. ${ }^{11,17}$ Vertical deviations interfere with fusion and their presence also indicates that the strabismus is of early onset. ${ }^{5}$ The resulting poor binocular vision could probably account for the exotropic change in alignment for esotropic patients with vertical deviations.

There was no difference between the change in alignment of exotropia patients with vertical deviations and exotropia patients without vertical deviations after 6 weeks and after $\geq 6$ months. According to Koo et al., vertical deviations had no effect on undercorrection in exotropia patients. ${ }^{16}$ In this study, the later age of onset of exotropia could probably account for better fusion despite the vertical deviation.

\section{Amblyopia}

There was no difference between the change in alignment of esotropia patients with amblyopia and esotropia patients without amblyopia. This is consistent with studies that showed that amblyopia was not a significant factor for surgical success of strabismus. ${ }^{11,14,15}$ This study excluded sensory deprivation strabismus patients. This accounted for less than severe amblyopia and therefore better fusional ability in the patients.

There was no difference between the change in alignment of exotropia patients with amblyopia and exotropia patients without amblyopia. Studies differ as to the effects of amblyopia on postoperative alignment in exotropia. ${ }^{10,16}$ The later onset and less frequent constant deviations of exotropia may account for the lesser influence of amblyopia on postoperative alignment.

\section{Preoperative deviation}

There was no difference between the change in alignment in esotropia patients with preoperative deviations <50 PD and patients with preoperative deviations $\geq 50 \mathrm{PD}$. This is consistent with the study of Keenan which showed that preoperative deviation had no effect on the surgical outcome of esotropia patients. ${ }^{15}$

For exotropia patients, there was a difference between the change in alignment of the group with a preoperative deviation $<50 \mathrm{PD}$ and the group with a preoperative deviation $\geq 50$ PD. Patients with larger preoperative deviations were more likely to have an esotropic change in alignment while those with <50 PD of exotropia preoperatively were more likely to have an exotropic change in alignment after 6 weeks. There was no difference between the change in alignment of the larger and smaller angle preoperative deviation groups after $\geq 6$ months. This study is consistent with the study of Koo et al. which showed that preoperative deviation is not a factor in postoperative undercorrection. ${ }^{16}$ Some studies however showed that a larger preoperative deviation was associated with a larger postoperative exotropic change in alignment. ${ }^{9}, 10$ Umazume et al. said that anatomical changes that normally take place in the muscle and fascia are more extensive in patients with larger preoperative deviations than in patients with smaller deviations. This difference may be related to differences in surgical outcome. ${ }^{22}$ In this study, preoperative deviation affected the change in alignment initially, however, the effect did not last over time in exotropia patients. After 6 weeks, those with larger preoperative deviations had an esotropic change in alignment probably due to the compensation for a higher frequency of undercorrection in larger angle exotropia. After 6 months however, the direction of the drift was towards exotropia.

\section{CONCLUSION AND RECOMMENDATION}

An esotropic drift was observed for overcorrected esotropia. An exotropic drift was observed for undercorrected esotropia. An esotropic drift was observed for undercorrected exotropia while an exotropic drift was observed for adequately corrected exotropia. Despite these changes in alignment, the patients were adequately corrected (deviation within 10 PD of orthotropia) at least 6 months after surgery. There was no correlation between the age at onset of strabismus or age at surgery and the change in alignment. The type of esotropia or exotropia and presence of amblyopia did not affect change in alignment. Although an exotropic drift is usually expected in patients who are not satisfactorily aligned after surgery, the study showed that fusional vergences are 
used to counteract inadequate alignment after surgery. This may account for the observed infrequent cases of secondary strabismus surgery in this institution. With the results of this study showing a change in postoperative alignment towards orthophoria, it is advisable to wait several months before planning secondary surgeries especially for general ophthalmologists. This however does not negate the need for a meticulous preoperative examination, preparation, surgical technique, and postoperative management to attain an adequate postoperative alignment. A postoperative target of orthotropia for esotropia surgery and minimal esotropia for exotropia surgery is still advised. This study is limited by the small number of subjects. Although trends were seen, strong conclusions could not be drawn. Some of the results of this study were also inconsistent with longer term studies. A longer follow-up period is recommended.

\section{Statement of Authorship}

All authors participated in the data collection and analysis, and approved the final version submitted.

\section{Author Disclosure}

All authors declared no conflicts of interest.

\section{Funding Source}

This paper was funded by the authors.

\section{REFERENCES}

1. Mills MD, Coats DK, Donahue SP, Wheeler DT. Strabismus surgery for adults: a report by the American Academy of Ophthalmology. Ophthalmology. 2004; 111(6):1255-62.

2. Beauchamp GR, Black BC, Coats DK, Enzenauer RW, Hutchinson AK, Saunders RA, et al. The management of strabismus in adults-I. Clinical characteristics and treatment. J AAPOS. 2003; 7(4):233-40.

3. Hertle RW. Clinical characteristics of surgically treated adult strabismus. J Pediatr Ophthalmol Strabismus. 1998; 35(3):138-45.

4. Raab EL. Difficult Esotropia Entities: Principles of Management. In: Rosenbaum A, Santiago AP, eds. Clinical strabismus management: Principles and surgical techniques. Philadelphia, PA: Saunders; 1999. pp. 139-151.

5. Von Noorden GK, Campos EC. Principles of Surgical Treatment. Binocular vision and ocular motility: Theory and management of strabismus. 6th ed. St. Louis, MO: Mosby; 2002. pp. 566-631.

6. Raab EL, Parks MM. Recession of the lateral recti: Effect of preoperative fusion and distance-near relationship. Arch Ophthalmol. 1975; 93(8):584-6.
7. Ruttum MS. Initial versus subsequent postoperative motor alignment in intermittent exotropia. J AAPOS. 1997; 1(2):88-91.

8. Pineles SL, Deitz LW, Velez FG. Postoperative outcomes of patients initially overcorrected for intermittent exotropia. J AAPOS. 2011; 15(6):527-31.

9. Yam JC, Chong GS, Wu PK, Wong US, Chan CW, Ko ST. Predictive factors affecting the short term and long term exodrift in patients with intermittent exotropia after bilateral rectus muscle recession and its effect on surgical outcome. BioMed Res Int. 2014; 2014:482093.

10. Park KH, Kim SY. Clinical characteristics of patients that experience different rates of exodrift after strabismus surgery for intermittent exotropia and the effect of the rate of exodrift on final ocular alignment. J AAPOS. 2013;17(1):54-8.

11. Mireskandari K, Schofield J, Cotesta M, Stephens D, Kraft SP. Achieving postoperative target range increases success of strabismus surgery in adults: A case for adjustable sutures. Br J Ophthalmol. 2015; 99(12):1697-701.

12. Chatzistefanou KI, Ladas ID, Droutas KD, Koutsandrea $\mathrm{C}$, Chimonidou E. Three horizontal muscle surgery for large-angle infantile or presumed infantile esotropia: Long-term motor outcomes. JAMA Ophthalmol. 2013; 131(8):1041-8.

13. Maruo T, Kubota N, Sakaue T, Usui C. Esotropia surgery in children: long term outcome regarding changes in binocular alignment; a study of 956 cases. Binocul Vis Strabismus Q. 2000; 15(3):213-20.

14. Keenan JM, Willshaw HE. The outcome of strabismus surgery in childhood exotropia. Eye. 1994; 8:632-7.

15. Keenan JM, Willshaw HE. The outcome of strabismus surgery in childhood esotropia. Eye. 1993; 7:341-5.

16. Koo NK, Lee YC, Lee SY. Clinical study for the undercorrection factor in intermittent exotropia. Korean J Ophthalmol. 2006; 20(3):182-7.

17. Maruo T, Kubota N, Iwashige H, Kamiya Y. Long-term results after strabismus surgery. Graefes Arch Clin Exp Ophthalmol. 1988; 226(5):414-7.

18. Kushner BJ, Fisher MR, Lucchese NJ, Morton GV. Factors influencing response to strabismus surgery. Arch Ophthalmol. 1993; 111(1):75-9.

19. Egbert JE, Fantin A. Factors influencing measurement and response to strabismus surgery. In: Rosenbaum A, Santiago AP, eds. Clinical strabismus management: Principles and surgical techniques. Philadelphia, PA: Saunders; 1999. pp. 73-83.

20. Paysse E, Coats D. Amblyopia. In: Nelson L, Olitsky S, eds. Harley's Pediatric Ophthalmology. 5th ed. Philadelphia, PA: Lippincott Williams and Wilkins; 2005.

21. Kothari M, Bhaskare A, Mete D, Toshniwal S, Doshi P, Kaul S. Evaluation of central, steady and maintained fixation grading for predicting inter-eye visual acuity difference to diagnose and treat amblyopia in strabismic patients. Indian J Ophthalmol. 2009; 57(4):281-4.

22. Umazume F, Ohtsuki H, Hasebe S. Preoperative factors influencing effectiveness of surgery in adult strabismus. Jpn J Ophthalmol. 1997; 41(2):89-97. 